\title{
Prognostic significance of phosphorylated signal transducer and activator of transcription 3 and suppressor of cytokine signaling 3 expression in hepatocellular carcinoma
}

\author{
WEN-YONG WU ${ }^{1,3}, \mathrm{JUN} \mathrm{LI}^{1}$, ZHENG-SHENG WU ${ }^{2}$, CHANG-LE ZHANG ${ }^{3}$, \\ XIANG-LING MENG ${ }^{3}$ and PETER E. LOBIE ${ }^{4}$
}

\begin{abstract}
${ }^{1}$ School of Pharmacy, and ${ }^{2}$ Department of Pathology, Anhui Medical University; ${ }^{3}$ Department of General Surgery, First Affiliated Hospital of Anhui Medical University, Hefei, Anhui, P.R. China; ${ }^{4}$ Cancer Science Institute of Singapore and Department of Pharmacology, National University of Singapore, Republic of Singapore
\end{abstract}

Received February 24, 2011; Accepted April 8, 2011

DOI: $10.3892 / \mathrm{etm} .2011 .254$

\begin{abstract}
Altered expression of phosphorylated signal transducer and activator of transcription 3 (pSTAT3) and suppressor of cytokine signaling 3 (SOCS3) has been implicated in various types of human cancers. However, the clinical role of pSTAT3 and SOCS3 in hepatocellular carcinoma (HCC) is not well established. Immunohistochemical analysis of pSTAT3, SOCS3, Ki67 and VEGF expression was performed on tissue microarrays from 138 HCC patients. The expression of STAT3 mRNA was further detected by in situ hybridization. The association of pSTAT3 and SOCS3 expression with clinicopathological factors and patient survival was analyzed. Altered expression of pSTAT3 and SOCS3 was observed in HCC specimens, compared to adjacent non-tumor tissue. Increased expression of pSTAT3 was correlated with large tumor size, higher clinical stage, Ki67 and VEGF expression, as well as poor patient survival. Decreased expression of SOCS3 was correlated with the expression of Ki67, VEGF and pSTAT3, and poor patient survival. Moreover, the expression of pSTAT3 was conversely correlated with SOCS3 expression in HCC. Our results indicate that deregulated expression of pSTAT3 and SOCS3 may play roles in the development and progression of HCC. PSTAT3 and SOCS3 should be further evaluated as potential novel biomarkers for HCC prognosis.
\end{abstract}

\section{Introduction}

Hepatocellular carcinoma (HCC) is one of the most common and aggressive human malignant neoplasias worldwide. Despite considerable diagnostic and therapeutic advances in recent

Correspondence to: Dr Jun Li, School of Pharmacy, Anhui Medical University, 81 Meishan Road, Hefei, Anhui 230032, P.R. China

E-mail: lijun@ahmu.edu.cn

Key words: hepatocellular carcinoma, phosphorylated signal transducer and activator of transcription 3 , suppressor of cytokine signaling 3 , prognosis years, the overall outcome of HCC patients remains dismal (1). To improve the effectiveness of diagnosis and therapy, and thus reduce mortality, it is crucial to develop novel biomarkers for early detection, for monitoring tumor progression and for predicting the prognosis of HCC.

The Janus family of tyrosine kinases (JAK) and the signal transducer and activator of transcription (STAT) family have been shown to play important roles in diverse signal transduction pathways which are involved in many biological processes, including cell proliferation, differentiation, survival and apoptosis, as well as cancer development and progression (2-4). Studies have also demonstrated a strong association between the expression of STAT proteins and the progression of various human malignances $(5,6)$. Among the STAT family members, STAT3 is the most commonly activated in human epithelial cancers (7-12). STAT3 is a latent cytoplasmic transcription factor which is tyrosine-phosphorylated by tyrosine kinase signals. Notably, STAT3 is constitutively activated, and contributes to oncogenesis and progression by directly or indirectly regulating the expression of genes required for cell proliferation, survival and angiogenesis (7). By contrast, suppressors of cytokine signaling (SOCS) have been implicated as negative regulators of cytokine signaling, including the JAK-STAT pathway (13). In particular, SOCS3 is regarded to be an endogenous inhibitor of STAT3 by suppression of its activation $(14,15)$. Although several studies have implicated their roles in a diverse group of human carcinomas, the relationship between STAT3 and SOCS3 in HCC remains poorly understood.

Herein, we determined the expression of pSTAT3 protein and STAT3 mRNA in 138 HCC and 110 adjacent non-tumorous hepatic tissue specimens on tissue microarray, and explored the correlation of pSTAT3 and SOCS3 expression in HCC with clinicopathologic features. We further demonstrated the relationship between pSTAT3 and SOCS3 expression in HCC. Therefore, the detection of pSTAT3 and SOCS3 expression may be helpful as a combined prognostic indicator in HCC.

\section{Materials and methods}

Patients and specimens. The patient population consisted of 138 consecutive HCC patients who underwent partial hepa- 
tectomy at the First Affiliated Hospital of Anhui Medical University (Hefei, Anhui, China) between 2004 and 2007. HCC patients who had undergone chemotherapy or radiation therapy before surgery were excluded, as were patients with rheumatic disease, acute infection, HIV or other types of cancer. The pathological tumor stage was defined according to the sixth edition of the tumor-node-metastasis (TNM) classification of the International Union against Cancer. Tumor differentiation was defined according to the Edmondson grading system (16). Complete follow-up data were obtained from all HCC patients. Primary study end points were post-operative overall survival (OS) and post-operative relapse-free survival (RFS). OS and RFS were defined as the time from the date of surgery to the date of death from HCC or to the date of local recurrence or detection of distant metastasis, respectively. All tissue diagnoses were confirmed by permanent histology. Institutional ethics committee approval for the project was granted before the study was commenced and was in compliance with the Helsinki Declaration. Written informed consent was obtained from all patients.

Tissue microarray (TMA) construction. Paraffin-embedded tumor and adjacent non-tumorous tissue specimens were obtained from the archive of the Department of Pathology, The First Affiliated Hospital of Anhui Medical University, China. We reviewed all the H\&E-stained sections from each paraffin-embedded, formalin-fixed block to identify target areas. Three to five representative 1-mm cores were obtained from each case and inserted in a grid pattern into a recipient paraffin block by using a tissue arrayer (Hengtai Instruments Inc., Liaoning, China).

Immunohistochemical analysis. We performed immunohistochemical analyses of pSTAT3, SOCS3, Ki67 and vascular endothelial growth factor (VEGF) protein expression in TMA sections $(3-\mu \mathrm{m})$ using a Two-Step histostaining kit (Maixin, Fuzhou, China) with monoclonal antibodies against human pSTAT3 (1:100; Santa Cruz Biotechnology, Inc., Santa Cruz, CA, USA), SOCS3 (1:200; Santa Cruz Biotechnology), Ki67 (working solution, Maixin) and VEGF (working solution, Maixin). The assay was performed as described previously (17). In brief, sections were deparaffinized in xylene, rehydrated in a graded series of ethanol solutions and heated in a microwave oven in $0.01 \mathrm{M}$ sodium citrate buffer $(\mathrm{pH} 6.0$ ) for $10 \mathrm{~min}$ for antigen retrieval. The sections were then immersed in $3 \%$ hydrogen peroxide in methanol for $10 \mathrm{~min}$ to block endogenous peroxidase activity. After rinsing with phosphate-buffered saline (PBS), the sections were incubated with primary antibodies in a moist chamber for $1 \mathrm{~h}$ at ambient temperature, and then incubated in horseradish peroxidaseconjugated secondary antibody for $20 \mathrm{~min}$. To visualize positive signals, the sections were incubated with 3,3'-diaminobenzidine solution for $5 \mathrm{~min}$ and then counterstained with hematoxylin solution, dehydrated and mounted. All experiments included separate known positive and negative controls. For the negative control samples, the primary antibody was replaced by PBS only.

In situ hybridization. For detecting the expression of STAT3 mRNA in HCC and adjacent non-tumorous tissues, an in situ hybridization assay was performed as previously described $(17,18)$. Briefly, 3- $\mu \mathrm{m}$ TMA sections were deparaffinized, rehydrated and then digested and refixed in $4 \%$ paraformaldehyde. The sections were then replaced with hybridization solution and incubated with the biotinylated oligonucleotide probe complementary to STAT3 mRNA (5'CCTTGGATTGAGAGTCAAGATTGGGCATAT3') (Boshide, Wuhan, China) and scrambled sequence (negative control) at $40^{\circ} \mathrm{C}$ for $20 \mathrm{~h}$. After washing, the sections were incubated with a mouse anti-digoxin antibody followed by binding to the streptavidin-biotin-peroxidase complex. Next, the sections were stained with 3,3'-diaminobenzidine solution and counterstained with a hematoxylin solution.

Scoring of stained sections. The stained sections were reviewed and scored for expression of pSTAT3, SOCS3, Ki67 and VEGF protein and STAT3 mRNA using an Olympus microscope (Olympus America, Inc., Melville, NY, USA) independently by two experienced pathologists who had no knowledge of the patient identities or clinical status. Positive staining for pSTAT3 expression was defined as $>25 \%$ nuclear staining with more than moderate intensity (8). Cytoplasmic staining in $>30 \%$ of cells was considered positive for SOCS3 (19), and cytoplasmic and/or membranous staining in $>50 \%$ of cells was considered positive for VEGF (8). The Ki67 labeling index was scored by counting 500 cells and determining the percentage of cells that stained positively for Ki67: score +, $0-25 \%$; score,$++ 26-50 \%$; score,$+++ 51-75 \%$; score ++++ , $>75 \%$ (20). For the expression of STAT3 mRNA, the core tissue was scored as positive when $>10 \%$ of the cytoplasm was stained.

Statistical analysis. All statistical analyses were performed using SPSS software system for Windows (version 13.0; SPSS, Chicago, IL, USA). Differences between groups were compared using the Pearson's Chi-square test for qualitative variables and the Student's t-test for continuous variables. Kaplan-Meier curves were constructed to determine patient RFS and OS rates. The statistical differences in survival among subgroups were compared using the log-rank test. $\mathrm{P}<0.05$ was considered statistically significant.

\section{Results}

Expression of pSTAT3 and SOCS3 in HCC and adjacent nontumorous tissue specimens. To determine the expression of pSTAT3 and SOCS3 in hepatic tissues, immunohistochemical staining was performed on $138 \mathrm{HCC}$ and 110 corresponding adjacent non-tumor tissues (at least $2 \mathrm{~cm}$ from the carcinoma margin). pSTAT3 protein was mainly expressed in the nucleus of tumor cells or non-tumorous hepatic cells, whereas immunoreactive SOCS3 protein was predominantly located in the cytoplasm of cells (Fig. 1). As shown in Table I, pSTAT3 protein expression was detected in 75 (54.3\%) of the HCC tissues and $35(35.8 \%)$ of the non-tumor tissue $(\mathrm{P}<0.001)$, while the expression of SOCS3 protein was significantly decreased in the HCC compared to that in the non-tumor tissue $(46.4$ and $72.7 \%, \mathrm{P}<0.001)$.

To further confirm the expression of pSTAT3 found in the immunohistochemical analysis, digoxigenin-labeled antisense oligonucleotide probe was utilized to detect STAT3 mRNA in 


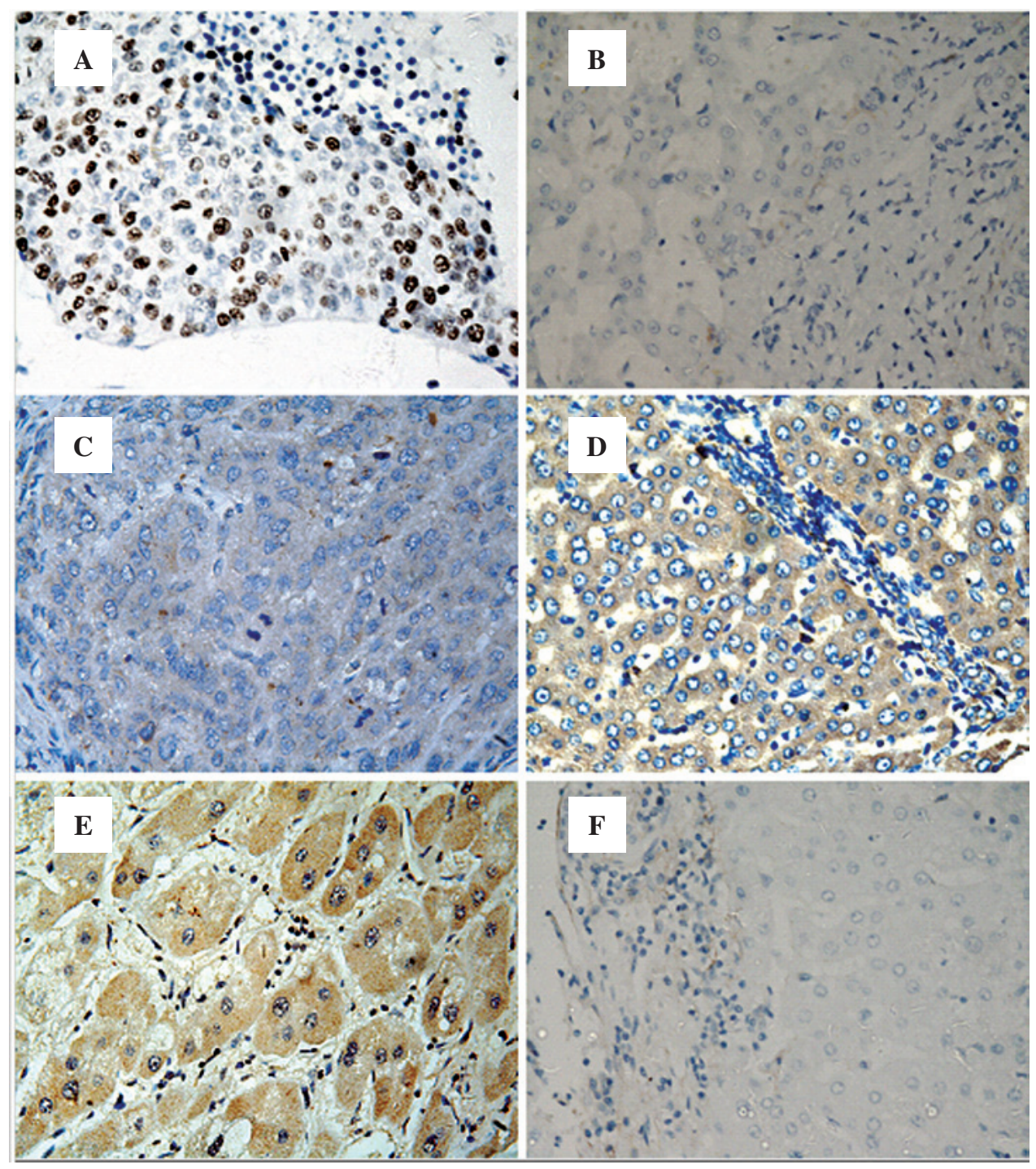

Figure 1. Immunohistochemical analysis of pSTAT3 and SOCS3 protein expression and in situ hybridization analysis of pSTAT3 mRNA expression in hepatocellular carcinoma (HCC) and adjacent non-tumor tissue. (A) pSTAT3 protein expression in HCC; (B) pSTAT3 protein expression in non-tumor tissue; (C) SOCS3 protein expression in HCC; (D) SOCS3 protein expression in non-tumor tissue; (E) STAT3 mRNA expression in HCC; (F) STAT3 mRNA expression in non-tumor tissue. All images were counterstained with hematoxylin. Original magnification, $\mathrm{x} 400$.

Table I. Expression of pSTAT3 and SOCS3 in the HCC and adjacent non-tumor tissue specimens.

\begin{tabular}{lcccc}
\hline & & \multicolumn{3}{c}{ Positive expression, $\mathrm{n}(\%)$} \\
\cline { 3 - 4 } Group & No. & pSTAT3 protein & SOCS3 protein & STAT3 mRNA \\
\hline HCC & 138 & $75(54.3)^{\mathrm{a}}$ & $64(46.4)^{\mathrm{a}}$ & $70(50.7)^{\mathrm{a}}$ \\
Non-tumorous & 110 & $35(31.8)$ & $80(72.7)$ & $33(30.0)$ \\
\hline
\end{tabular}

${ }^{\mathrm{a}} \mathrm{P}<0.001$.

the same cohort of hepatic tissue specimens. In situ hybridization staining revealed that the expression of STAT3 mRNA was located in the cytoplasm of cells (Fig. 1), and STAT3 mRNA expression was also significantly higher in the HCC than in the non-tumorous hepatic tissues (50.7 and $30.0 \%$, respectively; $\mathrm{P}<0.001)$. Furthermore, Spearman's correlation analyses revealed that the expression of STAT3 mRNA was significantly correlated with the expression of pSTAT3 protein (correlation coefficient, $\mathrm{r}_{\mathrm{s}}=0.763 ; \mathrm{P}<0.001$ ).

Correlation between the expression of pSTAT3 and SOCS3 protein. To detect the relationship between pSTAT3 and SOCS3, 
Table II. Correlation between the expression of pSTAT3 and SOCS3 in HCC tissues.

\begin{tabular}{lccc}
\hline & \multicolumn{2}{c}{ pSTAT3 } \\
\cline { 3 - 4 } & & $\begin{array}{c}\text { Negative, } \\
\text { no. }(\%)\end{array}$ & $\begin{array}{c}\text { Positive, } \\
\text { no. }(\%)\end{array}$ \\
\hline SOCS3 & Negative, n (\%) & $13(17.6)$ & $61(82.4)^{\mathrm{a}}$ \\
& Positive, n (\%) & $50(78.1)$ & $14(21.9)$ \\
\hline
\end{tabular}

${ }^{\mathrm{a}} \mathrm{P}<0.001$.

we performed Spearman's correlation analyses of protein expression in the HCC tissue specimens. As shown in Table II, SOCS3 protein expression was deficient in 74 of 138 HCC cases, in which the nuclear accumulation of pSTAT3 protein was detected in $61(82.4 \%)$. Among the 64 cases which expressed SOCS3 protein, only $14(21.9 \%)$ HCC cases exhibited stronger positivity for pSTAT3. Correlation analysis revealed that reduced SOCS3 protein expression was significantly correlated with the high expression of pSTAT3 protein $\left(\mathrm{r}_{\mathrm{s}}=-0.606, \mathrm{P}<0.001\right)$.
Association of the expression of pSTAT3 and SOCS3 with clinicopathological features of HCC. Next, we demonstrated the association of pSTAT3 and SOCS3 protein with the clinicopathological features of the HCC patients. As shown in Table III, pSTAT3 expression was associated with tumor size $(\mathrm{P}=0.001)$ and higher clinical stage $(\mathrm{P}=0.002)$. In addition, pSTAT3 expression was also correlated with the expression of proliferation-associated antigen Ki67 and the pro-angiogenic factor VEGF ( $\mathrm{P}=0.000$ and 0.001 , respectively). Furthermore, a trend was found for the decreased expression of SOCS3 and large tumor size and high clinical stage, although these did not reach significance $(\mathrm{P}=0.137$ and 0.251 , respectively). However, SOCS3 expression was conversely correlated with the expression of Ki67 and VEGF ( $\mathrm{P}=0.034$ and 0.001 , respectively).

Association of the expression of pSTAT3 and SOCS3 with patient survival. To determine whether the expression of pSTAT3 and SOCS3 is associated with OS and RFS of HCC patients, we performed Kaplan-Meier analyses. As shown in Fig. 2, patients whose primary tumors expressed pSTAT3 protein had a significantly poorer OS and RFS after curative resection compared to those without pSTAT3 expression $(\mathrm{P}=0.034$ and 0.001 , respectively). In addition, a significant

Table III. Association of pSTAT3 and SOCS3 expression with clinicopathological parameters of the HCC patients.

\begin{tabular}{|c|c|c|c|c|c|}
\hline Parameter & No. & pSTAT3 expression, $\mathrm{n}(\%)$ & P-value & SOCS3-positive expression, $\mathrm{n}(\%)$ & P-value \\
\hline Age (years) & & & 0.133 & & 0.967 \\
\hline$\leq 55$ & 86 & $51(59.3)$ & & $40(46.5)$ & \\
\hline$>55$ & 52 & $24(46.2)$ & & $24(46.2)$ & \\
\hline Gender & & & 0.252 & & 0.222 \\
\hline Male & 115 & $60(52.2)$ & & $56(48.7)$ & \\
\hline Female & 23 & $15(65.2)$ & & $8(34.8)$ & \\
\hline Cirrhosis & & & 0.533 & & 0.986 \\
\hline Yes & 125 & $69(55.2)$ & & $58(46.4)$ & \\
\hline No & 13 & $6(46.2)$ & & $6(46.2)$ & \\
\hline HBsAg & & & 0.888 & & 0.513 \\
\hline Yes & 111 & $60(54.1)$ & & $53(47.7)$ & \\
\hline No & 27 & 15 (55.6) & & $11(40.7)$ & \\
\hline Tumor size $(\mathrm{cm})$ & & & 0.001 & & 0.137 \\
\hline$<5$ & 41 & $13(31.7)$ & & $23(56.1)$ & \\
\hline$\geq 5$ & 97 & $62(63.9)$ & & $41(42.3)$ & \\
\hline Grade & & & 0.417 & & 0.299 \\
\hline I & 5 & $4(80.0)$ & & $4(80.0)$ & \\
\hline II & 128 & $69(53.9)$ & & $58(45.3)$ & \\
\hline III & 5 & $2(40.0)$ & & $2(40.0)$ & \\
\hline Stage & & & 0.002 & & 0.251 \\
\hline I-II & 106 & $50(47.2)$ & & $52(49.1)$ & \\
\hline III-IV & 32 & $25(78.1)$ & & $12(37.5)$ & \\
\hline Ki67 & & & 0.000 & & 0.034 \\
\hline$<50 \%$ & 60 & $22(36.7)$ & & $34(56.7)$ & \\
\hline$\geq 50 \%$ & 78 & $53(67.9)$ & & $30(38.5)$ & \\
\hline VEGF & & & 0.001 & & 0.001 \\
\hline Negative & 53 & 12 (22.6) & & $37(69.8)$ & \\
\hline Positive & 85 & $63(74.1)$ & & $27(31.8)$ & \\
\hline
\end{tabular}



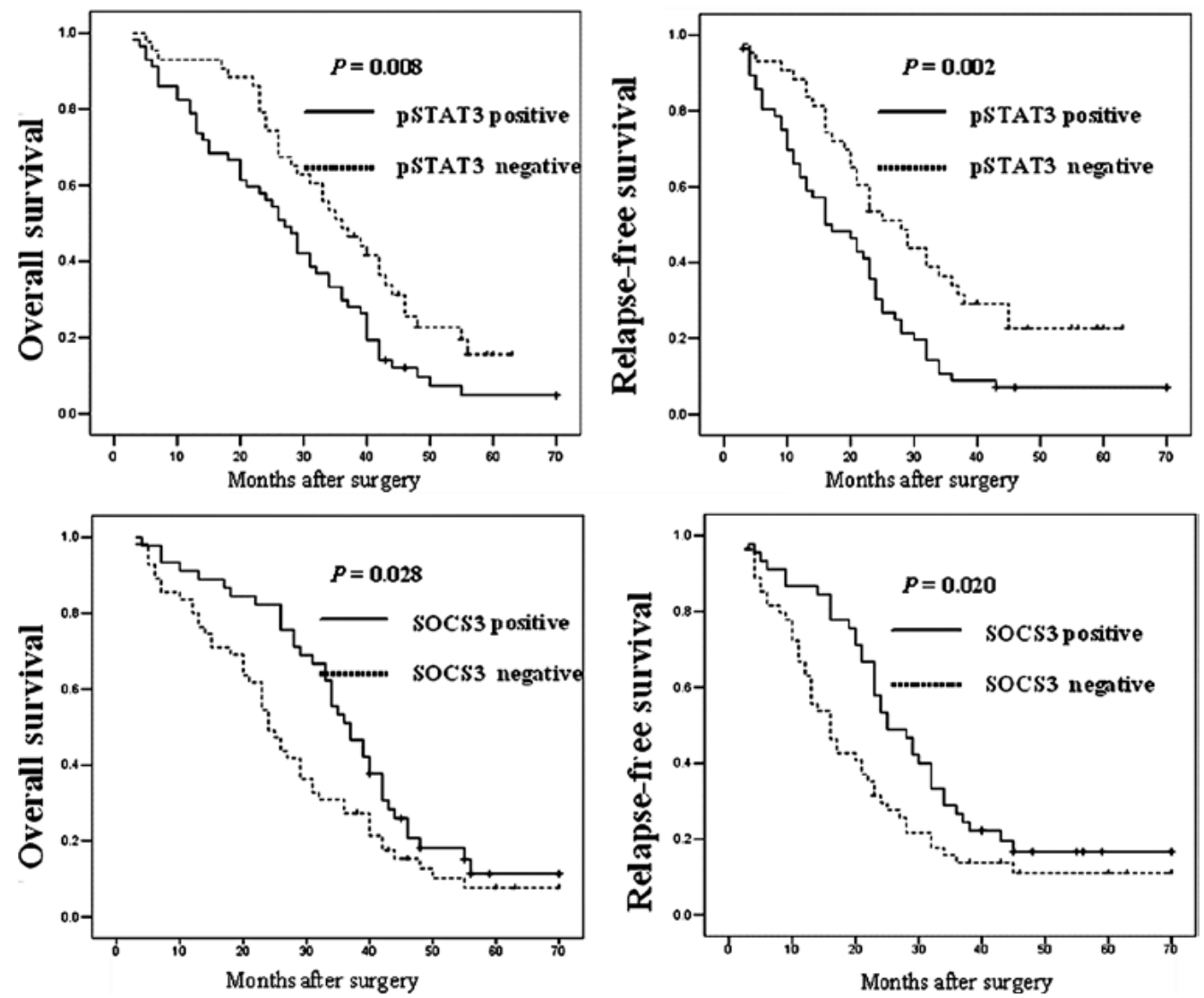

Figure 2. Kaplan-Meier analysis of pSTAT3 and SOCS3 protein expression in hepatocellular carcinoma patients.

association of SOCS3 protein expression with patient OS and RFS was found, which showed that patients with SOCS3positive expression had increased OS and RFS rates when compared with patients without SOCS3 expression $(\mathrm{P}=0.028$ and 0.020 , respectively).

\section{Discussion}

In the present study, the expression of pSTAT3 protein and STAT3 mRNA was significantly increased, while the expression of SOCS3 was decreased in HCC compared to adjacent non-tumor tissues. Furthermore, the expression of pSTAT3 and SOCS3 was conversely correlated in the HCC tissue specimens. In addition, high expression of pSTAT3 and low expression of SOCS3 protein were associated with disease progression and poor patient outcome. Our data offer clinical evidence that pSTAT3 and SOCS3 play fundamental roles in HCC development and progression. The data also suggest that pSTAT3 and SOCS3 may serve as potential biomarkers for predicting the prognosis of HCC patients.

The STAT family comprises seven members: STAT1-4, STAT5a, STAT5b and STAT6 (6). Since the STAT family regulates the expression of multiple genes involved in both physiological and pathological conditions, it seems to be one of the most promising biomarkers for predicting disease progression and the prognosis of patients with various cancer types. Among the STAT family members, activated STAT3 plays a vital role in a diverse group of human cancers. Yang et al (9) detected pSTAT3 expression in $69 \mathrm{HCC}$ and corresponding adjacent non-tumor parenchyma by immunohistochemistry, and found that $49.3 \%$ of $\mathrm{HCC}$ and $5.8 \%$ of non-tumorous tissues were positive for pSTAT3 nuclear staining. This observation differed quantitatively, but not qualitatively with our observation that pSTAT3-positive expression was observed in $54.3 \%$ of HCC and $31.8 \%$ of non-tumor tissues. This discrepancy may be attributed to the different population of patients, subjective interpretation by pathologists or assay achieving.

To avoid the limitations of immunohistochemistry, we also performed in situ hybridization for a more precise detection of STAT3 mRNA expression. The result of STAT3 mRNA expression detected from in situ hybridization was consistent with pSTAT3 protein expression. In other types of carcinomas, Deng et al (10) reported that the expression of both PSTAT3 protein and STAT3 mRNA was significantly higher in gastric cancer than in normal gastric tissues. They further demonstrated that the level of pSTAT3 immunohistochemical expression was positively associated with the status of lymph node metastasis and poor patient survival. Constitutive activation of STAT3 has also been implicated in lung cancer development. Kim et al (11) detected pSTAT3 and VEGFR1 expression by immmunohistochemistry and found that pSTAT3 was expressed with higher frequency (51.2\%) in 162 lung adenocarcinomas. Their data further revealed that the coexpression of pSTAT3 and VEGFR1 was significantly correlated with increased lymph node involvement and a trend towards a decreased OS. Similarly, in urothelial carcinoma, Huang et al (12) demonstrated that 
the expression of pSTAT3 protein was positively associated with tumor invasiveness and high histological grade. In our HCC patient population, increased pSTAT3 expression was observed, in accord with previous studies, and our data further demonstrated that pSTAT3 expression correlated with higher tumor stage and decreased patient survival. Moreover, pSTAT3 expression has been implicated in tumor cell proliferation, and tumoral VEGF production and angiogenesis $(8,21,22)$. Thus, we also observed a correlation between pSTAT3 expression and tumor expression of Ki67 and VEGF.

SOCS are a family of proteins that regulate negative feedback to the signaling cascade of JAK/STAT activating cytokine $(23,24)$. There are eight members of the SOCS family: the cytokine inducible Src homology 2 domaincontaining protein and SOCS1-7 (24). In the present study, decreased expression of SOCS3 was found to correlate with poor patient survival and expression of Ki67, VEGF and pSTAT3, suggesting a tumor suppressor role of SOCS3 in HCC. Indeed, the function of SOCS3 has been examined in vitro and in vivo as a negative regulator of STATs. Forced expression of SOCS3 was found to result in growth inhibition of human lung adenocarcinoma cells $(25,26)$. Conditional knockouts of SOCS3 displayed sustained interleukin (IL)-6mediated activation of STAT3, suggesting SOCS3 as a crucial inhibitor of STAT3 in vivo (27). However, contradictory reports exist concerning its role as a protector of tumor cells or tumor suppressor in studies using clinical specimens. Huang et al found that SOCS3 expression was higher in non-invasive urothelial carcinoma than in invasive urothelial carcinoma (12). Further study of breast carcinoma (28) showed that the expression of SOCS3 was significantly decreased in breast carcinoma cell lines and clinical specimens, compared to normal and adjacent non-tumor breast tissue. Their data further revealed that deficient expression of SOCS3 was significantly associated with lymph node metastasis, blood vessel invasion, expression of VEGF and Ki-67 and reduced disease-free survival, concordant with our study in HCC. Moreover, Nakagawa et al (29) demonstrated that decreased expression of SOCS3 mRNA was correlated with tumor lymph node metastasis in breast carcinoma. However, Yang et al (21) detected the expression of SOCS3 in 87 HCC patients and observed that $67.8 \%$ of HCC lesions showed moderate to very strong SOCS3 staining, and increased expression of SOCS3 was positively associated with tumor vascular invasion and poor patient OS. In addition, it has been reported that forced expression of SOCS3 protects chronic myelogenous leukaemia and cutaneous T-cell lymphoma against growth inhibition by IFN- $\alpha(30,31)$. These contradictions in the literature reflect the complex role of SOCS3 in different types of malignancies and require further investigation.

In general, our study suggests that altered expression of pSTAT3 and SOCS3 may play important roles in HCC development and progression. However, further studies are required to verify the usefulness of pSTAT3 and SOCS3 as a biomarker in different ethnic populations.

\section{Acknowledgements}

This study was supported, in part, by a grant from the Anhui Medical University.

\section{References}

1. He J, Gu D, Wu X, et al: Major causes of death among men and women in China. N Engl J Med 353: 1124-1134, 2005.

2. Hirano T, Ishihara K and Hibi M: Roles of STAT3 in mediating the cell growth, differentiation and survival signals relayed through the IL-6 family of cytokine receptors. Oncogene 19: 2548-2556, 2000.

3. Valentino L and Pierre J: JAK/STAT signal transduction: regulators and implication in hematological malignancies. Biochem Pharmacol 71: 713-721, 2006.

4. Buettner R, Mora LB and Jove R: Activated STAT signaling in human tumors provides novel molecular targets for therapeutic intervention. Clin Cancer Res 8: 945-954, 2002.

5. Bowman T, Garcia R, Turkson J and Jove R: STATs in oncogenesis. Oncogene 19: 2474-2488, 2000.

6. Yu H and Jove R: The STATs of cancer - new molecular targets come of age. Nat Rev Cancer 4: 97-105, 2004.

7. Kanda N, Seno H, Konda Y, et al: STAT3 is constitutively activated and supports cell survival in association with survivin expression in gastric cancer cells. Oncogene 23: 4921-4929, 2004.

8. Choi JH, Ahn MJ, Park CK, Han HX, Kwon SJ, Lee YY and Kim IS: Phospho-Stat3 expression and correlation with VEGF, $\mathrm{p} 53$, and $\mathrm{Bcl}-2$ in gastric carcinoma using tissue microarray. APMIS 114: 619-625, 2006

9. Yang SF, Wang SN, Wu CF, Yeh YT, Chai CY, Chunag SC, Sheen MC and Lee KT: Altered p-STAT3 (tyr705) expression is associated with histological grading and intratumour microvessel density in hepatocellular carcinoma. J Clin Pathol 60: 642-648, 2007.

10. Deng JY, Sun D, Liu XY, Pan Y and Liang H: STAT-3 correlates with lymph node metastasis and cell survival in gastric cancer. World J Gastroenterol 16: 5380-5387, 2010.

11. Kim HS, Park YH, Lee J, Ahn JS, Kim J, Shim YM, Kim JH, Park K, Han J and Ahn MJ: Clinical impact of phosphorylated signal transducer and activator of transcription 3, epidermal growth factor receptor, p53, and vascular endothelial growth factor receptor 1 expression in resected adenocarcinoma of lung by using tissue microarray. Cancer 116: 676-685, 2010.

12. Huang WT, Yang SF, Wu CC, Chen WT, Huang YC, Su YC and Chai CY: Expression of signal transducer and activator of transcription 3 and suppressor of cytokine signaling 3 in urothelial carcinoma. Kaohsiung J Med Sci 25: 640-646, 2009.

13. Kile BT and Alexander WS: The suppressors of cytokine signalling (SOCS). Cell Mol Life Sci 58: 1627-1635, 2001.

14. Bai L, Yu Z, Qian G, Qian P, Jiang J, Wang G and Bai C: SOCS3 was induced by hypoxia and suppressed STAT3 phosphorylation in pulmonary arterial smooth muscle cells. Respir Physiol Neurobiol 152: 83-91, 2006.

15. O'Shea JJ, Gadina M and Schreiber RD: Cytokine signaling in 2002: new surprises in the Jak/Stat pathway. Cell 109: S121-S131, 2002.

16. Edmondson HA and Steiner PE: Primary carcinoma of the liver: a study of 100 cases among 48, 900 necropsies. Cancer 7: 462-503, 1954.

17. Wu ZS, Wu Q, Yang JH, Wang HQ, Ding XD, Yang F and $\mathrm{Xu}$ XC: Prognostic significance of MMP-9 and TIMP-1 serum and tissue expression in breast cancer. Int J Cancer 122: 2050-2056, 2008.

18. Wu ZS, Wu Q, Wang CQ, Wang XN, Wang Y, Zhao JJ, Mao SS, Zhang GH, Zhang N and Xu XC: MiR-339-5p inhibits breast cancer cell migration and invasion in vitro and may be a potential biomarker for breast cancer prognosis. BMC Cancer 10: 542, 2010.

19. Li Y, de Haar C, Chen M, Deuring J, Gerrits MM, Smits R, Xia B, Kuipers EJ and van der Woude CJ: Disease-related expression of the IL6/STAT3/SOCS3 signalling pathway in ulcerative colitis and ulcerative colitis-related carcinogenesis. Gut 59: 227-235, 2010.

20. Brown DC and Gatter KC: Ki67 protein: the immaculate deception? Histopathology 40: 2-11, 2002.

21. Yang SF, Yeh YT, Wang SN, Hung SC, Chen WT, Huang $\mathrm{CH}$ and Chai CY: SOCS-3 is associated with vascular invasion and overall survival in hepatocellular carcinoma. Pathology 40: 558-563, 2008.

22. Niu G, Wright KL, Huang M, et al: Constitutive Stat3 activity up-regulates VEGF expression and tumor angiogenesis. Oncogene 21: 2000-2008, 2002. 
23. Alexander WS and Hilton DJ: The role of suppressors of cytokine signaling (SOCS) proteins in regulation of the immune response. Annu Rev Immunol 22: 503-529, 2004.

24. Yoshimura A, Nishinakamura H, Matsumura Y and Hanada T: Negative regulation of cytokine signaling and immune responses by SOCS proteins. Arthritis Res Ther 7: 100-110, 2005.

25. He B, You L, Uematsu K, Zang K, Xu Z, Lee AY, Costello JF, McCormick F and Jablons DM: SOCS-3 is frequently silenced by hypermethylation and suppresses cell growth in human lung cancer. Proc Natl Acad Sci USA 100: 14133-14138, 2003.

26. Yu ZB, Bai L, Qian P, Xiao YB, Wang GS, Qian GS, Bai CX and Min JX: Restoration of SOCS3 suppresses human lung adenocarcinoma cell growth by downregulating activation of Erk1/2, Akt apart from STAT3. Cell Biol Int 33: 995-1001, 2009.

27. Croker BA, Krebs DL, Zhang JG, et al: SOCS3 negatively regulates IL-6 signaling in vivo. Nat Immunol 4: 540-545, 2003.
28. Ying M, Li D, Yang L, Wang M, Wang N, Chen Y, He M and Wang Y: Loss of SOCS3 expression is associated with an increased risk of recurrent disease in breast carcinoma. J Cancer Res Clin Oncol 136: 1617-1626, 2010.

29. Nakagawa T, Iida S, Osanai T, Uetake H, Aruga T, Toriya Y, Takagi Y, Kawachi $\mathrm{H}$ and Sugihara K: Decreased expression of SOCS-3 mRNA in breast cancer with lymph node metastasis. Oncol Rep 19: 33-39, 2008.

30. Sakai I, Takeuchi K, Yamauchi H, Narumi H and Fujita S: Constitutive expression of SOCS3 confers resistance to IFN-alpha in chronic myelogenous leukemia cells. Blood 100: 2926-2931, 2002.

31. Brender C, Lovato P, Sommer VH, Woetmann A, Mathiesen AM, Geisler C, Wasik $M$ and Ødum N: Constitutive SOCS-3 expression protects T-cell lymphoma against growth inhibition by IFNalpha. Leukemia 19: 209-213, 2005. 